\section{Mudanças de comportamentos relacionados à saúde como preditores de mudanças na autopercepção de saúde: estudo longitudinal (2011-2015)}

\author{
Changes in health-related behaviors as predictors \\ of changes in health self-perception: Iongitudinal \\ study (2011-2015)
}

\section{Cambios de comportamientos relacionados con la salud como predictores de cambios en la autopercepción de la salud: estudio longitudinal (2011-2015)}

Giovana Frazon de Andrade 1

Mathias Roberto Loch 1

Ana Maria Rigo Silva 1

doi: 10.1590/0102-311X00151418

\title{
Resumo
}

A autopercepção de saúde é reconhecida como um interessante indicador de saúde, inclusive em função da sua capacidade preditora de morbimortalidade. Estudos que investigam sua associação com comportamentos relacionados à saúde são mais recentes e, em sua maioria, transversais, indicando poder haver melhor compreensão dessa relação em estudos longitudinais. O objetivo deste artigo foi verificar a relação entre mudanças nos comportamentos relacionados à saúde e a incidência de autopercepção positiva e negativa de saúde. Foi realizado um estudo de coorte prospectivo, de base populacional, com 883 indivíduos de quarenta anos ou mais, entrevistados em 2011 e 2015. Verificou-se a incidência de autopercepção negativa e positiva de saúde segundo mudanças em quatro comportamentos relacionados à saúde: atividade física no tempo livre, consumo de frutas e hortaliças, consumo abusivo de álcool e tabagismo - por meio da regressão de Poisson bruta e ajustada. A incidência de autopercepção negativa de saúde foi de 27,2\%, sendo maior, após ajustes com variáveis demográficas, de saúde e demais comportamentos, entre os indivíduos que apresentaram mudança negativa na atividade física no tempo livre $(R R=1,88$; IC95\%: 1,17-3,05) e no consumo de frutas e hortaliças $(R R=$ 1,95; IC95\%: 1,15-3,28). A incidência de autopercepção positiva de saúde foi $33,2 \%$, sendo superior nos que tiveram mudança positiva no tabagismo $(R R=$ 8,37; IC95\%: 2,79-25,09) e inferior nos que apresentaram mudança negativa no consumo de frutas e hortaliças ( $R R=0,51$; IC95\%: 0,29-0,90). Concluise que algumas modificações nos comportamentos relacionados à saúde têm um possível impacto na autopercepção da saúde, o que reforça a importância de politicas públicas relativas aos comportamentos relacionados à saúde, indo para além da sua relação com a morbimortalidade.

Estilo de Vida; Comportamentos Relacionados com a Saúde; Autoavaliação; Indicadores Básicos de Saúde; Estudos Longitudinais

\section{Correspondência}

G. F. Andrade

Programa de Pós-graduação em Ciências da Saúde, Universidade Estadual de Londrina.

Av. Robert Kock 60, Londrina, PR 86038-350, Brasil. giovanafrazon@hotmail.com

1 Universidade Estadual de Londrina, Londrina, Brasil. 


\section{Introdução}

Incorporada como medida epidemiológica a partir da década de 1950, a autopercepção de saúde tem sido um indicador de saúde bastante utilizado, inclusive por sua fácil aplicação, por ter boa validade e confiabilidade 1 e por ser um bom preditor de morbimortalidade 2,3. Ademais, a autopercepção de saúde tem sido relacionada a aspectos demográficos, sociais e de saúde, demonstrando as diferenças existentes entre os grupos populacionais e auxiliando na identificação de necessidades prioritárias em saúde e suas tendências 4,5. A relação entre comportamentos de risco à saúde e a presença de pior autopercepção de saúde tem sido evidenciada em diversos estudos transversais 1,6,7,8.

Investigar essa associação é relevante, tendo em vista que a promoção de comportamentos saudáveis tem sido indicada como uma importante questão de saúde pública, sendo incorporada, no contexto brasileiro, por diversas políticas públicas e programas, entre os quais o Plano Nacional de Promoção da Saúde (PNPS) 9, a Política Nacional de Atenção Básica (PNAB) 10, o Plano de Enfrentamento das Doenças Crônicas Não Transmissíveis 11 e o Programa Academia da Saúde 12. Entre os principais comportamentos considerados de risco à saúde estão a inatividade física 13,14, o baixo consumo de frutas e hortaliças 15,16, o consumo abusivo de álcool 17,18 e o tabagismo 19 .

As pesquisas conduzidas no Brasil para investigar a relação entre comportamentos de saúde e autopercepção de saúde são, em sua maioria, de delineamento transversal, sendo este possivelmente o primeiro estudo brasileiro que aborda essa relação a partir de um desenho longitudinal. No contexto internacional, os estudos sobre autopercepção de saúde que utilizam esse delineamento também são escassos 20 . Assim, o objetivo deste estudo foi verificar a relação entre mudanças em determinados comportamentos relacionados à saúde e à incidência de autopercepção positiva e negativa de saúde após quatro anos de acompanhamento em indivíduos de quarenta anos ou mais residentes em um município de médio porte da Região Sul do Brasil.

\section{Métodos}

Trata-se de estudo observacional longitudinal prospectivo, realizado nos anos de 2011 e 2015, cuja população foi constituída por indivíduos com idade igual ou superior a quarenta anos, residentes na zona urbana do Município de Cambé, região metropolitana de Londrina, norte do Estado do Paraná. No baseline do estudo, em 2011, partiu-se de uma amostra populacional definida a partir da contagem da população realizada em 2007 (92.888 pessoas) pelo Instituto Brasileiro de Geografia e Estatística (IBGE; http://ibge.gov.br). Desse quantitativo, 30.710 pessoas $(33,1 \%)$ estavam na faixa etária do estudo. A amostra foi calculada pelo aplicativo StatCalc (http://www.ucs.louisiana.edu/ kxk4695/ StatCalc.htm), do programa Epi Info 3.5.3 (https://www.cdc.gov/epiinfo/index.html), considerando-se prevalência do desfecho de 50\%, margem de erro de $3 \%$ e intervalo de $95 \%$ de confiança (IC95\%), totalizando 1.066 indivíduos. Prevendo-se perdas e recusas, foram acrescentados $25 \%$ a esse número, totalizando 1.332 indivíduos. Considerou-se, ainda, a distribuição proporcional entre homens e mulheres acima de quarenta anos para cada um dos 86 setores censitários da zona urbana do município, com arredondamento dos cálculos, ficando a amostra final com 1.339 indivíduos. Para seleção dos entrevistados, inicialmente os bairros, as ruas e as quadras foram identificados em mapas dos setores censitários da zona urbana da cidade. As quadras foram numeradas, e foram sorteados os pontos iniciais da coleta (quadra, esquina e residência). A partir desse ponto, o percurso seguia sentido anti-horário, elegendo-se domicílios de forma alternada. Em cada domicílio visitado, eram identificados os moradores de quarenta anos ou mais elegíveis para a entrevista e, nos casos de mais de um residente atender às condições determinadas, sorteava-se apenas um deles. Como critérios de exclusão, consideraram-se indivíduos que apresentavam deficiência visual e/ou auditiva graves, dificuldade no entendimento dos procedimentos da entrevista por não compreensão do idioma português e transtornos mentais em estágio avançado.

Ocorreram 159 perdas (11,9\%), 93 recusas e 66 indivíduos não encontrados após três tentativas. Assim, em 2011, foram entrevistados 1.180 indivíduos. Mais informações sobre o processo de amostragem e características dos indivíduos em 2011 estão detalhadas no artigo de Souza et al. 21. 
Em 2015, todos os sujeitos entrevistados em 2011, localizados e com consentimento, participaram do seguimento do estudo. As entrevistas eram previamente agendadas pelo telefone informado no baseline. Quando não era possível o agendamento por telefone, eram realizadas visitas domiciliares, e apenas se considerava perda após três tentativas de procura em dias e horários diferentes. Nessa etapa, ocorreram 295 perdas (25\%). Dentre elas, houve 51 óbitos e 87 recusas. Além disso, 49 não foram encontrados após três tentativas e 108 mudaram de endereço. Houve ainda duas perdas referentes aos sujeitos que não responderam a questão sobre autopercepção de saúde em 2015, totalizando 883 indivíduos que participaram de ambos os anos e com dados completos da variável de desfecho.

Em 2011 e 2015, a equipe de entrevistadores foi composta de alunos de graduação e pós-graduação, do Centro de Ciências da Saúde da Universidade Estadual de Londrina, e de profissionais da área da saúde. Todos os entrevistadores passaram por treinamento prévio. Em 2011, foi realizado estudo piloto em um bairro de cidade vizinha para teste e correções do instrumento de coleta e treinamento dos entrevistadores.

As entrevistas duraram, em média, 30 minutos em 2011 e 40 minutos em 2015. No momento da entrevista, era apresentado ao participante o Termo de Consentimento Livre e Esclarecido (TCLE) e era realizada a aplicação do questionário. Também, nesse mesmo momento, eram feitas três medidas da pressão arterial e aferição de peso e estatura. Após o término da entrevista, agendava-se data e local para coleta de sangue. Ambos os projetos (2011 e 2015) foram aprovados pelo Comitê de Ética em Pesquisa envolvendo Seres Humanos, Universidade Estadual de Londrina, Londrina, em 2010 (CAAE no 0192.0.268.000-10) e em 2014 (CAAE no 39595614.4.0000.5231), respectivamente.

\section{Descrição das variáveis}

A variável dependente foi a autopercepção de saúde, aplicando-se a mesma pergunta do estudo de Vigilância de Fatores e Risco e Proteção para Doenças Crônicas por Inquérito Telefônico (VIGITEL) do Ministério da Saúde 22: “Como o(a) senhor(a) classifica seu estado de saúde?”. As opções de resposta eram: "muito bom; bom; regular; ruim; muito ruim". Optou-se pela dicotomização em autopercepção positiva de saúde (APPS, para os indivíduos que responderam "muito bom e bom") e autopercepção negativa de saúde (APNS, para os indivíduos que responderam "regular, ruim e muito ruim"). Para trabalhar a mudança entre 2011 e 2015, considerou-se a condição inicial dos indivíduos, sendo que os que apresentavam APNS no baseline $(\mathrm{n}=397)$ foram categorizados em manutenção da APNS e incidentes de APPS (mudança positiva). Por sua vez, indivíduos que apresentavam APPS no baseline $(\mathrm{n}=486)$ foram categorizados em manutenção da APPS e incidentes da APNS (mudança negativa).

As variáveis independentes, avaliadas com as mesmas perguntas em 2011 e em 2015, foram os seguintes comportamentos relacionados à saúde: atividade física no tempo livre, consumo de frutas e/ou hortaliças, consumo abusivo de álcool e tabagismo. Para a atividade física no tempo livre, foi utilizada a seguinte pergunta: "Em uma semana normal (típica), o(a) Sr.(a) faz algum tipo de atividade física no seu tempo livre?". As opções de resposta eram "sim" (fisicamente ativo no tempo livre) e "não" (fisicamente inativo no tempo livre). Os demais comportamentos também foram investigados com base nas questões utilizadas pelo VIGITEL 22. Para o consumo de frutas e hortaliças, foram utilizadas duas perguntas. A primeira era a seguinte: "Em quantos dias da semana o(a) Sr.(a) costuma comer frutas?". As opções de resposta eram "nunca; quase nunca; 1-2 dias; 3-4 dias; 5-6 dias; todos os dias". A segunda pergunta era: "Em quantos dias da semana o(a) $\mathrm{Sr}$.(a) costuma comer pelo menos um tipo de verdura ou legume (alface, tomate, couve, chuchu, berinjela, abobrinha), sem contar batata, mandioca ou inhame?”. As opções de resposta eram "nunca; quase nunca; 1-2 dias; 3-4 dias; 5-6 dias; todos os dias". Realizou-se a dicotomização das respostas, considerando-se como "consumo regular" os indivíduos que consumiam frutas cinco dias ou mais por semana, e "consumo irregular" os demais, sendo o mesmo critério adotado para o consumo de hortaliças (verduras ou legumes). Após essa dicotomização, houve a junção das duas variáveis, e foram considerados com "consumo regular" de frutas e hortaliças os indivíduos que consumiam regularmente tanto frutas como hortaliças. $\mathrm{O}$ "consumo irregular", por sua vez, indicava aqueles que consumiam irregularmente frutas e/ou hortaliças. Para o consumo abusivo de álcool, foi utilizada a seguinte pergunta: "Nos últimos 30 dias, o(a) Sr.(a) consumiu mais que quatro (para mulheres) ou cinco (para homens) doses de bebida alcoólica em uma ocasião?". As opções de resposta eram "sim" (considerado como consumo abusivo de álcool) e "não" 
(considerado como consumo não abusivo de álcool). Para o tabagismo, utilizou-se a pergunta "O(a) Sr.(a) fuma?", com as opções de resposta "sim” (tabagista) e "não" (não tabagista).

Para o ajuste, foram utilizadas variáveis sociodemográficas do baseline: sexo (feminino e masculino), faixa etária (40-49 anos, 50-59 anos e $\geq 60$ anos) e escolaridade (0-4 anos, 5-8 anos e 9 anos ou mais). Também foram utilizadas outras variáveis, considerando a mudança da condição entre 2011 e 2015: situação conjugal (mantiveram-se com companheiro(a); deixaram de ter companheiro(a); mantiveram-se sem companheiro(a); passaram a ter companheiro(a)), classe econômica 23 (mantiveram-se com nível econômico A ou B; eram da classe $\mathrm{AB}$ e passaram a ser da classe $\mathrm{C}$, D ou E; mantiveram-se na classe econômica C, D ou E; eram da classe econômica C, D ou E e passaram a ser AB), doenças autorreferidas (indivíduos que mantiveram ou diminuíram o número de doenças autorreferidas; indivíduos que aumentaram o número de doenças autorreferidas) e mudança no estado nutricional (baixo peso ou eutrofia nos dois momentos; tinham baixo peso/eutrofia em 2011 e passaram a ter sobrepeso/ obesidade em 2015; sobrepeso/obesidade nos dois momentos; tinham sobrepeso/obesidade em 2011 e passaram a ter baixo peso/eutrofia em 2015). Vale mencionar que o cálculo do estado nutricional foi realizado por meio do índice de massa corporal (IMC), e aplicou-se a classificação do estado nutricional, por faixa etária, utilizada pelo Sistema de Vigilância Alimentar e Nutricional (SISVAN) do Ministério da Saúde (http://dabsistemas.saude.gov.br/sistemas/sisvanV2/).

\section{Tabulação e análise de dados}

As informações obtidas no questionário físico, em ambas as coletas de dados, foram duplamente digitadas em banco de dados do programa Microsoft Office Excel 2010 (https://products.office.com/) e comparadas através do software Epi Info versão 3.5.3 (no ano de 2011) e software Microsoft Office SpreadSheet Compare (no ano de 2015). No ano de 2015, informações também foram coletadas em formulário eletrônico do ODK Collect (Open Data Kit), que eram enviadas pela Internet e armazenadas no servidor ONA e posteriormente exportadas para banco de dados do programa Microsoft Office Excel. As análises estatísticas foram realizadas pelo programa Statistical Package for the Social Sciences - SPSS - versão 19.0 para Windows (https://www.ibm.com/). As características dos respondentes e perdas, assim como a incidência de APNS e APPS segundo mudança nas variáveis socioeconômicas e de saúde, foram apresentadas por meio de distribuição de frequências absolutas e relativas. A comparação das características entre os respondentes e perdas se deu pelo teste qui-quadrado de Pearson, considerando-se $\mathrm{p}<0,05$. A incidência de APNS e APPS relacionada às mudanças nos comportamentos em saúde foi calculada por tabulação cruzada entre as variáveis, gerando distribuição de frequências absolutas e relativas. Foi calculado o risco relativo (RR) pela regressão de Poisson, com variância robusta, bruta e ajustada. Foi realizada ainda regressão linear, e não foi constatada colinearidade entre as variáveis independentes.

A análise de regressão foi realizada comparando-se a incidência de APPS e APNS (variáveis dependentes), conforme manutenção ou mudança de cada comportamento investigado (variáveis independentes). Foi considerada como estratégia a estratificação em relação a cada uma das variáveis independentes, considerando as informações do baseline. Definiu-se que o grupo referência seria sempre aquele que se manteve com o comportamento nas duas coletas, com manutenção do comportamento positivo ou negativo. Por exemplo: no caso da inatividade física no tempo livre, inicialmente foram selecionados somente os sujeitos que eram ativos em 2011 e comparou-se a incidência de APNS e de APPS daqueles que permaneceram ativos (grupo referência) com a daqueles que passaram a ser inativos (ou seja, tiveram uma mudança negativa nesse comportamento). A mesma lógica foi repetida para os demais comportamentos e também com a mudança de comportamento "positiva" (isto é, comparando-se a incidência de APPS e APNS daqueles que passaram a ter o comportamento considerado positivo em $2015 \mathrm{com}$ a daqueles que se mantiveram com o comportamento considerado negativo).

O cálculo do risco relativo foi ajustado por três diferentes modelos. No modelo 1, foi realizado o ajuste a partir das seguintes variáveis: sexo, faixa etária, escolaridade, mudança ou manutenção na situação conjugal e no nível econômico. O modelo 2 foi ajustado pelas mesmas variáveis do modelo 1 , mais a manutenção ou mudança no estado nutricional e no número de doenças autorreferidas. $\mathrm{O}$ modelo 3 foi ajustado pelas variáveis dos modelos 1 e 2, mais as mudanças nos comportamentos relacionados à saúde (exceto, evidentemente, aquele comportamento que estava sendo analisado como 
variável independente). Por exemplo, quando o tabagismo era a variável independente, considerava-se no ajuste a manutenção ou a modificação nos demais comportamentos como variáveis de confusão, sendo possíveis quatro categorias para cada uma dessas variáveis (manutenção do comportamento positivo; mudança positiva entre o baseline e o seguimento; mudança negativa entre o baseline e o seguimento; manutenção do comportamento negativo). Outros modelos de ajustes foram testados, com a inclusão de variáveis do baseline (situação conjugal, nível econômico, doenças autorreferidas, estado nutricional e comportamentos relacionados à saúde), porém não apresentaram diferenças significativas em relação ao modelo 3 , de modo que se optou pela manutenção desse modelo.

\section{Resultados}

A amostra total deste estudo $(\mathrm{n}=883)$ foi composta majoritariamente de mulheres $(55,9 \%)$, indivíduos com faixa etária entre 40-49 anos (39,3\%) e situação conjugal com companheiro $(74,3 \%)$. Quanto à escolaridade e ao nível econômico, aproximadamente metade (47,7\%) tinha entre 0-4 anos de estudo, e a maioria era de classe econômica C ou inferior (62,1\%). Em relação à autopercepção de saúde e aos comportamentos relacionados à saúde, 55\% tinham APPS, 69,6\% eram totalmente inativos no tempo livre, $60,7 \%$ tinham consumo irregular de frutas e hortaliças, 18,8\% referiram consumo abusivo de álcool nos últimos 30 dias e 18,8\% eram tabagistas (Tabela 1). Comparando-se as características da amostra em $2011 \mathrm{com}$ as perdas, constatou-se maior percentual de perdas entre os indivíduos com sessenta anos ou mais, com APPS, que não realizavam atividade física no tempo livre (inativos), com consumo irregular de frutas e hortaliças e que eram tabagistas (Tabela 1).

A incidência total de APNS foi de $27,2 \%$, sendo maior no sexo feminino (30,3\%), na faixa etária entre 50-59 anos (35,1\%), com 0-4 anos de estudo (36,8\%), que se mantiveram sem companheiro (38,9\%), no nível econômico $\operatorname{CDE}(32,6 \%)$, que aumentaram o número de doenças autorreferidas $(27,9 \%)$ e que passaram a ter um estado nutricional de baixo peso/eutrófico (37,5\%). Quanto à APPS de saúde, a incidência total foi de $33,2 \%$, sendo superior no sexo masculino (38\%), na faixa etária entre 40-49 anos (40,2\%), com nove ou mais anos de estudo (51,4\%), que passaram a ter companheiro (37,5\%), que se mantiveram na classe $\mathrm{AB}(50 \%)$ ou diminuíram para $\mathrm{CDE}(50 \%)$ em 2015, que diminuíram ou mantiveram o número de doenças autorreferidas $(36,6 \%)$ e que passaram à classificação sobrepeso/obesidade (39,3\%) (Tabela 2).

Os indivíduos que apresentaram mudança negativa da atividade física no tempo livre (Tabela 3) tiveram maior incidência de APNS na análise bruta (RR = 1,74; IC95\%: 1,08-2,80) quando comparados àqueles que se mantiveram fisicamente ativos, mantendo-se maior após ajustes do primeiro $(\mathrm{RR}=$ 1,65; IC95\%: 1,04-2,62), do segundo ( $R R=1,92$; IC95\%: 1,19-3,10) e do terceiro modelos ( $R R=1,88$; IC95\%: 1,17-3,05). Os indivíduos que tiveram mudança negativa no consumo de frutas e hortaliças demonstraram maior incidência de APNS na análise bruta (RR = 1,84; IC95\%: 1,16-2,92), quando comparados àqueles que mantiveram o consumo regular de frutas e hortaliças, nos três modelos de ajuste avaliados: modelo $1(\mathrm{RR}=1,85$; IC95\%: 1,14-3,01), modelo 2 ( $\mathrm{RR}=2,03$; IC95\%: 1,25-3,30) e modelo $3(\mathrm{RR}=1,95$; IC95\%: 1,15-3,28). Já os indivíduos que apresentaram mudança positiva no consumo abusivo de álcool tiveram maior incidência de APNS na análise bruta $(R R=2,29$; IC95\%: 1,03-5,08), comparados àqueles que se mantiveram com consumo abusivo de álcool, mantendo sua associação apenas quando ajustada pelo modelo $2(\mathrm{RR}=2,42$; IC95\%: 1,12-5,25).

$\mathrm{Na}$ Tabela 4, verificou-se que pessoas que tiveram mudança positiva na atividade física no tempo livre apresentaram maior incidência de APPS na análise bruta (RR = 1,48; IC95\%: 1,03-2,15), comparadas àquelas que se mantiveram inativas, mantendo-se essa associação após ajuste das variáveis do modelo 1 ( $R R=1,47$; IC95\%: 1,02-2,13). Quando avaliado o consumo de frutas e hortaliças, verificou-se que os que apresentaram mudança negativa no consumo de frutas e hortaliças tiveram menor incidência de APPS no primeiro ( $R R=0,52$; IC95\%: 0,29-0,94), no segundo ( $R R=0,54$; IC95\%: 0,31$0,97)$ e no terceiro modelo ajustado ( $R R=0,51$; IC95\%: 0,29-0,90), comparados aos que se mantiveram com um consumo regular de frutas e hortaliças, e aqueles que apresentaram mudança positiva no tabagismo tiveram maior incidência de APPS no primeiro ( $R R=2,58$; IC95\%: 1,16-5,71), no segundo $(\mathrm{RR}=5,78$; IC95\%: 2,18-15,36) e no terceiro modelo de ajuste (RR = 8,37; IC95\%: 2,79-25,09), comparados àqueles que se mantiveram tabagistas entre 2011 e 2015. 
Tabela 1

Características sociodemográficas, de autopercepção de saúde e de comportamentos relacionados à saúde em 2011 dos respondentes e perdas. População de 40 anos ou mais residente em Cambé, Paraná, Brasil.

\begin{tabular}{|c|c|c|c|c|c|}
\hline \multirow[t]{2}{*}{ Variáveis * } & \multicolumn{2}{|c|}{ Respondentes } & \multicolumn{2}{|c|}{ Perdas } & \multirow[t]{2}{*}{ Valor de $p$ ** } \\
\hline & $\mathbf{n}$ & $\%$ & $\mathbf{n}$ & $\%$ & \\
\hline Total & 883 & 74,9 & 297 & 25,1 & \\
\hline Sexo & & & & & 0,06 \\
\hline Feminino & 494 & 55,9 & 148 & 49,8 & \\
\hline Masculino & 389 & 44,1 & 149 & 50,2 & \\
\hline Faixa etária (anos) & & & & & $<0,05$ \\
\hline $40-49$ & 347 & 39,3 & 129 & 43,4 & \\
\hline $50-59$ & 297 & 33,6 & 69 & 23,2 & \\
\hline 60 ou mais & 239 & 27,1 & 99 & 33,3 & \\
\hline Escolaridade (anos) & & & & & 0,6 \\
\hline 9 ou mais & 237 & 26,8 & 83 & 27,9 & \\
\hline $5-8$ & 225 & 25,5 & 67 & 22,6 & \\
\hline $0-4$ & 421 & 47,7 & 147 & 49,5 & \\
\hline Situação conjugal *** & & & & & 0,12 \\
\hline Com companheiro & 656 & 74,3 & 207 & 69,7 & \\
\hline Sem companheiro & 227 & 25,7 & 89 & 30,3 & \\
\hline Nível econômico \# & & & & & 0,38 \\
\hline$A-B$ & 334 & 37,9 & 115 & 38,7 & \\
\hline C & 466 & 52,8 & 147 & 49,5 & \\
\hline D-E & 82 & 9,3 & 35 & 11,8 & \\
\hline Autopercepção de saúde \#\# & & & & & $<0,05$ \\
\hline Positiva & 486 & 55,0 & 186 & 64,1 & \\
\hline Negativa & 397 & 45,0 & 104 & 35,9 & \\
\hline Atividade física no tempo livre $* \star \star$ & & & & & $<0,05$ \\
\hline Ativo & 268 & 30,4 & 69 & 23,3 & \\
\hline Inativo & 615 & 69,6 & 227 & 76,7 & \\
\hline 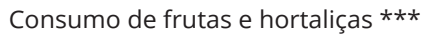 & & & & & $<0,05$ \\
\hline Regular & 347 & 39,3 & 96 & 32,4 & \\
\hline Irregular & 536 & 60,7 & 200 & 67,6 & \\
\hline Consumo abusivo de álcool \#\#\# & & & & & 0,12 \\
\hline Não & 716 & 81,5 & 252 & 85,4 & \\
\hline Sim & 163 & 18,5 & 43 & 14,6 & \\
\hline Tabagismo & & & & & $<0,05$ \\
\hline Não & 720 & 81,5 & 224 & 75,4 & \\
\hline Sim & 163 & 18,5 & 73 & 24,6 & \\
\hline
\end{tabular}

* Dados do baseline utilizados na análise;

** Valor de $p$ calculado pelo teste qui-quadrado de Pearson com $p<0,05$;

*** Um indivíduo com informação ausente nas perdas;

\# Um indivíduo com informação ausente nos respondentes;

\#\# Seis indivíduos com informação ausente nas perdas;

\#\#\# Dois indivíduos com informação ausente nos respondentes e dois indivíduos com informação ausente nas perdas. 


\section{Tabela 2}

Incidência de autopercepção negativa de saúde (APNS) e autopercepção positiva de saúde (APPS) segundo características demográficas e mudanças na situação conjugal, nível econômico, doenças autorreferidas e estado nutricional (mudanças entre 2011 e 2015). População de 40 anos ou mais residente em Cambé, Paraná, Brasil.

\begin{tabular}{|c|c|c|c|c|}
\hline \multirow[t]{2}{*}{ Variável } & \multicolumn{2}{|c|}{ Incidência de APNS ( $n=486)$} & \multicolumn{2}{|c|}{ Incidência de APPS ( $n=397)$} \\
\hline & $\mathbf{n}$ & $\%$ & $\mathbf{n}$ & $\%$ \\
\hline Total & 132 & 27,2 & 132 & 33,2 \\
\hline \multicolumn{5}{|l|}{ Sexo } \\
\hline Feminino & 74 & 30,3 & 75 & 30,4 \\
\hline Masculino & 58 & 24,0 & 57 & 38,0 \\
\hline \multicolumn{5}{|l|}{ Faixa etária (anos) } \\
\hline $40-49$ & 42 & 19,5 & 53 & 40,2 \\
\hline $50-59$ & 52 & 35,1 & 44 & 29,5 \\
\hline 60 ou mais & 38 & 30,9 & 35 & 30,2 \\
\hline \multicolumn{5}{|l|}{ Escolaridade (anos) } \\
\hline 9 ou mais & 31 & 19,0 & 38 & 51,4 \\
\hline $5-8$ & 27 & 22,1 & 37 & 35,9 \\
\hline $0-4$ & 74 & 36,8 & 57 & 25,9 \\
\hline \multicolumn{5}{|l|}{ Situação conjugal * } \\
\hline Manteve-se com companheiro & 76 & 23,2 & 85 & 33,2 \\
\hline Deixou de ter companheiro & 11 & 33,3 & 11 & 28,2 \\
\hline Manteve-se sem companheiro & 42 & 38,9 & 33 & 35,1 \\
\hline Passou a ter companheiro & 2 & 12,5 & 3 & 37,5 \\
\hline \multicolumn{5}{|l|}{ Nível econômico ** } \\
\hline Manteve-se AB & 31 & 20,8 & 36 & 50,0 \\
\hline $\mathrm{AB} \rightarrow \mathrm{CDE}$ & 15 & 26,3 & 28 & 50,0 \\
\hline Manteve-se CDE & 73 & 32,6 & 59 & 25,5 \\
\hline $\mathrm{CDE} \rightarrow \mathrm{AB}$ & 13 & 23,6 & 8 & 22,9 \\
\hline \multicolumn{5}{|l|}{ Doenças autorreferidas } \\
\hline Aumentou o número de doenças & 62 & 27,9 & 58 & 29,7 \\
\hline Diminuiu/manteve o número de doenças & 70 & 26,5 & 74 & 36,6 \\
\hline \multicolumn{5}{|l|}{ 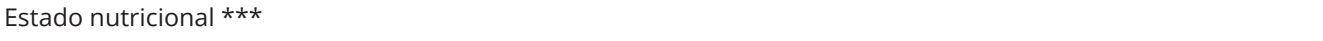 } \\
\hline Manteve-se baixo peso/eutrófico & 35 & 22,0 & 34 & 35,1 \\
\hline Baixo peso/eutrófico $\rightarrow$ Sobrepeso/obeso & 2 & 7,7 & 11 & 39,3 \\
\hline Manteve-se sobrepeso/obeso & 77 & 29,8 & 75 & 31,6 \\
\hline Sobrepeso/obeso $\rightarrow$ Baixo peso/eutrófico & 12 & 37,5 & 7 & 26,9 \\
\hline
\end{tabular}

Nota: dados do baseline utilizados nas variáveis fixas (sexo, faixa etária e escolaridade).

* Um indivíduo com informação ausente para APNS;

** Um indivíduo com informação ausente para APPS;

*** Seis indivíduos com informação ausente para APNS e cinco indivíduos com informação ausente para APPS.

\section{Discussão}

Em síntese, este estudo evidenciou que algumas modificações nos comportamentos relacionados à saúde influenciaram mudanças na autopercepção de saúde entre 2011 e 2015, sendo observada maior incidência de APNS entre indivíduos que fizeram a mudança negativa da atividade física no tempo livre e do consumo de frutas e hortaliças. Quanto à incidência de APPS, foi maior entre os que fizeram a mudança positiva do tabagismo e menor entre os que fizeram mudança negativa no consumo de frutas e hortaliças. 
Tabela 3

Incidência de autopercepção negativa de saúde (APNS) segundo mudanças nos comportamentos relacionados à saúde e respectivos riscos relativos (RR). População de 40 anos ou mais residente em Cambé, Paraná, Brasil $(n=486)$.

\begin{tabular}{|c|c|c|c|c|c|c|}
\hline Variáveis & Casos incidentes & $\%$ & $\begin{array}{c}\text { RR } \\
\text { (IC95\%) }\end{array}$ & $\begin{array}{c}\text { Modelo } 1 \\
\text { RR } \\
\text { (IC95\%) }\end{array}$ & $\begin{array}{c}\text { Modelo } 2 \\
\text { RR } \\
\text { (IC95\%) }\end{array}$ & $\begin{array}{c}\text { Modelo } 3 \\
\text { RR } \\
\text { (IC95\%) }\end{array}$ \\
\hline \multicolumn{7}{|c|}{ Mudança negativa da atividade física no tempo livre } \\
\hline Manteve-se ativo & 21 & 21,6 & 1,00 & 1,00 & 1,00 & 1,00 \\
\hline Ativo $\rightarrow$ Inativo & 29 & 37,7 & $\begin{array}{c}1,74 \\
(1,08-2,80)\end{array}$ & $\begin{array}{c}1,65 \\
(1,04-2,62)\end{array}$ & $\begin{array}{c}1,92 \\
(1,19-3,10)\end{array}$ & $\begin{array}{c}1,88 \\
(1,17-3,05)\end{array}$ \\
\hline \multicolumn{7}{|c|}{ Mudança positiva da atividade física no tempo livre } \\
\hline Manteve-se inativo & 70 & 28,1 & 1,00 & 1,00 & 1,00 & 1,00 \\
\hline Inativo $\rightarrow$ Ativo & 12 & 19,0 & $\begin{array}{c}0,68 \\
(0,39-1,17)\end{array}$ & $\begin{array}{c}0,72 \\
(0,41-1,25)\end{array}$ & $\begin{array}{c}0,73 \\
(0,41-1,33)\end{array}$ & $\begin{array}{c}0,77 \\
(0,43-1,37)\end{array}$ \\
\hline \multicolumn{7}{|c|}{ Mudança negativa do consumo de frutas e hortaliças } \\
\hline Manteve-se regular & 35 & 21,7 & 1,00 & 1,00 & 1,00 & 1,00 \\
\hline Regular $\rightarrow$ Irregular & 18 & 40,0 & $\begin{array}{c}1,84 \\
(1,16-2,92)\end{array}$ & $\begin{array}{c}1,85 \\
(1,14-3,01)\end{array}$ & $\begin{array}{c}2,03 \\
(1,25-3,30)\end{array}$ & $\begin{array}{c}1,95 \\
(1,15-3,28)\end{array}$ \\
\hline \multicolumn{7}{|c|}{ Mudança positiva do consumo de frutas e hortaliças } \\
\hline Manteve-se irregular & 56 & 28,1 & 1,00 & 1,00 & 1,00 & 1,00 \\
\hline Irregular $\rightarrow$ Regular & 23 & 28,4 & $\begin{array}{c}1,01 \\
(0,67-1,52)\end{array}$ & $\begin{array}{c}0,99 \\
(0,65-1,51)\end{array}$ & $\begin{array}{c}1,02 \\
(0,66-1,56)\end{array}$ & $\begin{array}{c}0,99 \\
(0,65-1,51)\end{array}$ \\
\hline \multicolumn{7}{|c|}{ Mudança negativa do consumo abusivo de álcool } \\
\hline Manteve-se não & 99 & 27,8 & 1,00 & 1,00 & 1,00 & 1,00 \\
\hline Não $\rightarrow \operatorname{Sim}$ & 7 & 30,4 & $\begin{array}{c}1,09 \\
(0,58-2,08)\end{array}$ & $\begin{array}{c}1,39 \\
(0,70-2,75)\end{array}$ & $\begin{array}{c}1,56 \\
(0,79-3,07)\end{array}$ & $\begin{array}{c}1,53 \\
(0,77-3,03)\end{array}$ \\
\hline \multicolumn{7}{|c|}{ Mudança positiva do consumo abusivo de álcool } \\
\hline Manteve-se sim & 7 & 13,7 & 1,00 & 1,00 & 1,00 & 1,00 \\
\hline $\operatorname{Sim} \rightarrow$ Não & 16 & 31,4 & $\begin{array}{c}2,29 \\
(1,03-5,08)\end{array}$ & $\begin{array}{c}2,21 \\
(0,98-5,01)\end{array}$ & $\begin{array}{c}2,42 \\
(1,12-5,25)\end{array}$ & $\begin{array}{c}2,72 \\
(0,89-8,28)\end{array}$ \\
\hline \multicolumn{7}{|c|}{ Mudança negativa do tabagismo } \\
\hline Manteve-se não & 107 & 27,2 & 1,00 & 1,00 & 1,00 & 1,00 \\
\hline Não $\rightarrow \operatorname{Sim}$ & 0 & 0,0 & - & - & - & - \\
\hline \multicolumn{7}{|c|}{ Mudança positiva do tabagismo } \\
\hline Manteve-se sim & 19 & 27,5 & 1,00 & 1,00 & 1,00 & 1,00 \\
\hline $\mathrm{Sim} \rightarrow$ Não & 6 & 30,0 & $\begin{array}{c}1,09 \\
(0,50-2,36)\end{array}$ & $\begin{array}{c}1,70 \\
(0,65-4,50)\end{array}$ & $\begin{array}{c}1,86 \\
(0,66-5,20)\end{array}$ & $\begin{array}{c}2,16 \\
(0,73-6,44)\end{array}$ \\
\hline
\end{tabular}

IC95\%: intervalo de 95\% de confiança.

Nota: RR calculado por regressão de Poisson. Modelo 1: ajustado por sexo, faixa etária, escolaridade, mudança no estado civil e mudança no nível econômico. Modelo 2: ajustado por sexo, faixa etária, escolaridade, mudança no estado civil, mudança no nível econômico, mudança no estado nutricional (índice de massa corporal) e mudança nas doenças autorreferidas. Modelo 3: sexo, faixa etária, escolaridade, mudança no estado civil, mudança no nível econômico, mudança no estado nutricional (índice de massa corporal), mudança nas doenças autorreferidas e comportamentos relacionados à saúde (atividade física no tempo livre, consumo de frutas e hortaliças, consumo abusivo de álcool, e tabagismo).

Em geral, existem poucos estudos longitudinais que realizam a análise da autopercepção de saúde como variável dependente/desfecho. Além disso, os estudos identificados apresentam diferentes pontos de corte na categorização da autopercepção de saúde, dificultando sua comparação. Esta pesquisa utilizou-se da questão sobre autopercepção de saúde inicialmente com cinco categorias de resposta e, posteriormente, as categorias foram dicotomizadas em APPS (muito bom, bom) e APNS (regular, ruim, muito ruim). No caso do VIGITEL 24, por exemplo, o relatório mais recente disponível (de 2016) aponta que 4,4\% tinham autopercepção de saúde ruim ou muito ruim, ou seja, o corte realizado pelo VIGITEL indicou a categoria "regular" como sendo positiva, ao contrário deste estudo. É importante mencionar que a inclusão das subcategorias "regular", "ruim" e "muito ruim" na 


\section{Tabela 4}

Incidência de autopercepção positiva de saúde (APPS) segundo mudanças nos comportamentos relacionados à saúde e respectivos riscos relativos (RR). População de 40 anos ou mais residente em Cambé, Paraná, Brasil $(n=397)$.

\begin{tabular}{|c|c|c|c|c|c|c|}
\hline Variáveis & Casos incidentes & $\%$ & $\begin{array}{c}\text { RR } \\
\text { (IC95\%) }\end{array}$ & $\begin{array}{c}\text { Modelo } 1 \\
\text { RR } \\
\text { (IC95\%) }\end{array}$ & $\begin{array}{c}\text { Modelo } 2 \\
\text { RR } \\
\text { (IC95\%) }\end{array}$ & $\begin{array}{c}\text { Modelo } 3 \\
\text { RR } \\
\text { (IC95\%) }\end{array}$ \\
\hline \multicolumn{7}{|c|}{ Mudança negativa da atividade física no tempo livre } \\
\hline Manteve-se ativo & 14 & 34,1 & 1,00 & 1,00 & 1,00 & 1,00 \\
\hline Ativo $\rightarrow$ Inativo & 20 & 37,7 & $\begin{array}{c}1,10 \\
(0,64-1,91)\end{array}$ & $\begin{array}{c}1,05 \\
(0,60-1,83)\end{array}$ & $\begin{array}{c}1,08 \\
(0,59-1,98)\end{array}$ & $\begin{array}{c}1,14 \\
(0,57-2,30)\end{array}$ \\
\hline \multicolumn{7}{|c|}{ Mudança positiva da atividade física no tempo livre } \\
\hline Manteve-se inativo & 77 & 30,1 & 1,00 & 1,00 & 1,00 & 1,00 \\
\hline Inativo $\rightarrow$ Ativo & 21 & 44,7 & $\begin{array}{c}1,48 \\
(1,03-2,15)\end{array}$ & $\begin{array}{c}1,47 \\
(1,02-2,13)\end{array}$ & $\begin{array}{c}1,43 \\
(0,98-2,08)\end{array}$ & $\begin{array}{c}1,33 \\
(0,87-2,02)\end{array}$ \\
\hline \multicolumn{7}{|c|}{ Mudança negativa do consumo de frutas e hortaliças } \\
\hline Manteve-se regular & 40 & 40,8 & 1,00 & 1,00 & 1,00 & 1,00 \\
\hline Regular $\rightarrow$ Irregular & 10 & 23,8 & $\begin{array}{c}0,58 \\
(0,32-1,05)\end{array}$ & $\begin{array}{c}0,52 \\
(0,29-0,94)\end{array}$ & $\begin{array}{c}0,54 \\
(0,31-0,97)\end{array}$ & $\begin{array}{c}0,51 \\
(0,29-0,90)\end{array}$ \\
\hline \multicolumn{7}{|c|}{ Mudança positiva do consumo de frutas e hortaliças } \\
\hline Manteve-se irregular & 57 & 31,3 & 1,00 & 1,00 & 1,00 & 1,00 \\
\hline Irregular $\rightarrow$ Regular & 25 & 33,3 & $\begin{array}{c}1,06 \\
(0,72-1,56)\end{array}$ & $\begin{array}{c}1,01 \\
(0,67-1,53)\end{array}$ & $\begin{array}{c}1,00 \\
(0,66-1,52)\end{array}$ & $\begin{array}{c}0,94 \\
(0,60-1,49)\end{array}$ \\
\hline \multicolumn{7}{|c|}{ Mudança negativa do consumo abusivo de álcool } \\
\hline Manteve-se não & 103 & 31,9 & 1,00 & 1,00 & 1,00 & 1,00 \\
\hline Não $\rightarrow$ Sim & 5 & 41,7 & $\begin{array}{c}1,31 \\
(0,66-2,60)\end{array}$ & $\begin{array}{c}1,23 \\
(0,62-2,43)\end{array}$ & $\begin{array}{c}1,17 \\
(0,57-2,40)\end{array}$ & $\begin{array}{c}1,23 \\
(0,56-2,70)\end{array}$ \\
\hline \multicolumn{7}{|c|}{ Mudança positiva do consumo abusivo de álcool } \\
\hline Manteve-se sim & 10 & 37,0 & 1,00 & 1,00 & 1,00 & 1,00 \\
\hline $\mathrm{Sim} \rightarrow$ Não & 14 & 42,4 & $\begin{array}{c}1,14 \\
(0,61-2,16)\end{array}$ & $\begin{array}{c}1,17 \\
(0,60-2,26)\end{array}$ & $\begin{array}{c}1,38 \\
(0,70-2,70)\end{array}$ & $\begin{array}{c}1,57 \\
(0,79-3,13)\end{array}$ \\
\hline \multicolumn{7}{|c|}{ Mudança negativa do tabagismo } \\
\hline Manteve-se não & 105 & 32,9 & 1,00 & 1,00 & 1,00 & 1,00 \\
\hline Não $\rightarrow$ Sim & 2 & 50,0 & $\begin{array}{c}1,52 \\
(0,56-4,10)\end{array}$ & $\begin{array}{c}1,39 \\
(0,60-3,17)\end{array}$ & $\begin{array}{c}1,42 \\
(0,63-3,18)\end{array}$ & $\begin{array}{c}1,46 \\
(0,53-4,02)\end{array}$ \\
\hline \multicolumn{7}{|c|}{ Mudança positiva do tabagismo } \\
\hline Manteve-se sim & 18 & 30,5 & 1,00 & 1,00 & 1,00 & 1,00 \\
\hline $\mathrm{Sim} \rightarrow$ Não & 7 & 46,7 & $\begin{array}{c}1,53 \\
(0,79-2,97)\end{array}$ & $\begin{array}{c}2,58 \\
(1,16-5,71)\end{array}$ & $\begin{array}{c}5,78 \\
(2,18-15,36)\end{array}$ & $\begin{array}{c}8,37 \\
(2,79-25,09)\end{array}$ \\
\hline
\end{tabular}

IC95\%: intervalo de 95\% de confiança.

Nota: RR calculado por Regressão de Poisson. Modelo 1: ajustado por sexo, faixa etária, escolaridade, mudança no estado civil e mudança no nível econômico. Modelo 2: ajustado por sexo, faixa etária, escolaridade, mudança no estado civil, mudança no nível econômico, mudança no estado nutricional (índice de massa corporal) e mudança nas doenças autorreferidas. Modelo 3: sexo, faixa etária, escolaridade, mudança no estado civil, mudança no nível econômico, mudança no estado nutricional (índice de massa corporal), mudança nas doenças autorreferidas e comportamentos relacionados à saúde (atividade física no tempo livre, consumo de frutas e hortaliças, consumo abusivo de álcool, e tabagismo).

categoria de APNS, no presente estudo, foi definida por ser a forma mais usual verificada na literatura 6,25,26,27,28. De qualquer modo, não é objetivo deste trabalho realizar comparações exaustivas com a incidência e a prevalência de APNS e APPS observadas em outros estudos.

Em relação às mudanças dos comportamentos avaliados nesta pesquisa, observou-se que a mudança negativa na atividade física no tempo livre aumentou a incidência de APNS, resultado que corrobora o obtido no estudo longitudinal realizado na Austrália, em que a manutenção da prática de atividade física associou-se ao menor declínio na APNS 20. Alguns estudos transversais também 
apontaram a relação entre a prática de atividade física e a maior prevalência de APPS, ou a relação entre menor prática de atividade física e piores níveis de autopercepção de saúde 7,25,26,28. A prática de atividade física traz potencialmente importantes benefícios à saúde, reduz a incidência de doenças crônicas e tende a aumentar a sensação de bem-estar físico e mental e qualidade de vida 29 , o que pode levar os indivíduos a uma melhor autopercepção de saúde. Além disso, fatores sociais podem perpassar os biológicos, visto que atividade física pode gerar relações sociais entre os indivíduos, como verificado por Loch et al. 27, constatando esse tipo de atividade como um importante mediador entre indicadores de capital social e autopercepção de saúde. Dados como intensidade, tipo e frequência da atividade física realizada no tempo livre pelo indivíduo não foram analisados, o que pode explicar a não associação entre tornar-se ativo e incidência de APPS, após ajuste nos modelos 2 e 3. Sugere-se que estudos futuros sobre a relação entre a atividade física no tempo livre e as mudanças na autopercepção de saúde incluam essas variáveis na sua análise.

A mudança negativa no consumo de frutas e hortaliças aumentou a incidência de APNS, e a manutenção do consumo regular associou-se à incidência de APPS, resultados que reforçam o que a literatura evidencia 7,30. Segundo o Guia Alimentar para a População Brasileira 16, o estudo dos efeitos individuais dos nutrientes se torna insuficiente na relação entre alimentação e saúde, sendo considerados outros aspectos relevantes que influenciam a saúde e o bem-estar dos indivíduos, como questões de combinação e preparo dos alimentos, modos de comer e também dimensões culturais e sociais das práticas alimentares. É possível analisar a alimentação por diferentes pontos de vista (por exemplo, econômico, nutricional e social), e esses mesmos pontos reunidos revelam importante influência na determinação do tipo de consumo alimentar do indivíduo. Um estilo de vida saudável inclui cuidados com o consumo alimentar, o que muitas vezes provoca mudanças significativas na relação do indivíduo com a comida, e isso se reflete na saúde de forma objetiva, como a melhora na vitalidade, e subjetiva, retratada na APPS. Na via contrária, a adoção de comportamentos alimentares não saudáveis, como o consumo irregular de frutas e hortaliças, atua no aumento dos riscos à saúde, sendo importante causa para o desenvolvimento de doenças crônicas não transmissíveis, entre elas, o sobrepeso e a obesidade 16,31, tornando possível a explicação da avaliação negativa de saúde, visto o aumento do risco relativo no modelo ajustado por fatores sociodemográficos e de saúde. Não foram coletadas informações mais completas sobre a alimentação, como a variedade e a quantidade, porém o consumo regular de frutas e hortaliças tem sido muito utilizado como um proxy de alimentação saudável 16.

Nesta pesquisa, não foram encontradas associações entre a mudança positiva no consumo de frutas e hortaliças e APNS e APPS, ou seja, assim como no caso da atividade física no tempo livre, parece que ter deixado de ter o comportamento considerado positivo exerceu maior influência na mudança da autopercepção de saúde do que a adoção do comportamento positivo propriamente dito (no caso, começar a fazer atividade física no tempo livre e passar a consumir regularmente frutas e hortaliças). É possível que a questão da temporalidade seja um dos aspectos que explique esses achados. Talvez haja a necessidade de um maior tempo de exposição aos comportamentos considerados saudáveis para que aconteçam modificações mais evidentes na autopercepção de saúde. Não temos, no presente estudo, a informação sobre o momento exato em que ocorreram as mudanças nos comportamentos relacionados à saúde e, de fato, obter essa precisão na medida é muito difícil em estudos longitudinais. Talvez estudos experimentais possam ter um maior controle nessa questão e possam fornecer mais informações sobre o tempo necessário para que a mudança nos hábitos relacionados à prática de atividade física ou de alimentação possa influenciar a autopercepção de saúde, mesmo que, evidentemente, deva existir grande variação individual nessa mudança.

Não foram encontradas associações entre mudança positiva no consumo abusivo de álcool com a APPS, assim como entre a mudança negativa do consumo abusivo de álcool e a APNS e APPS, possivelmente, em boa parte, pelo tamanho da amostra utilizada nessas análises. Mesmo não tendo sido encontradas associações significativas entre mudanças na exposição ao consumo abusivo de álcool e mudanças na autopercepção de saúde no último modelo de análise (ajustado por variáveis demográficas, de saúde e para os demais comportamentos relacionados à saúde), chamou a atenção a associação observada no modelo 2 (ajustado por variáveis demográficas e de saúde) e a APNS. Assim, ao contrário das demais associações significativas encontradas neste estudo, no caso do consumo abusivo de álcool, observou-se que deixar de consumir abusivamente aumentou a incidência de APNS. Esse resultado reforça a complexidade dessa relação e o quanto a autopercepção de saúde pode ser 
influenciada por questões que vão muito além dos aspectos biológicos, pois, pelo menos em teoria, se esperava que deixar de consumir álcool de maneira abusiva diminuiria a incidência de APNS, já que os malefícios do consumo abusivo de bebidas alcoólicas à saúde são apontados pela literatura, com destaque para o câncer, doenças hepáticas, distúrbios psiquiátricos, doenças cardiovasculares, acidentes de trânsito, intoxicações, violências, entre outros 32 . Outra hipótese que não pode ser descartada neste estudo é que algumas pessoas tenham parado de consumir álcool de maneira abusiva em função de outra condição negativa de saúde. Os ajustes utilizados, mudança do estado nutricional e mudança no número de doenças podem ter sido insuficientes para controlar esse viés.

Por outro lado, não se pode desconsiderar que o uso do álcool é frequentemente associado a fatores positivos na sociedade, inclusive em função do forte papel que a publicidade exerce, pelo menos no contexto brasileiro, na construção de um imaginário coletivo que associa o consumo de álcool a ambientes festivos, esportivos e culturais, entre outros. Assim, os determinantes sociais do consumo de álcool tornam-se complexos de serem analisados, pois o contexto cultural em que ele se insere, muitas vezes, estimula seu consumo $33,34,35$.

Outro fato a ser destacado é que a medida utilizada nesta pesquisa foi o consumo abusivo de álcool, medida que tem sido comumente usada na literatura 17,18,24, e não de dependência ao álcool. Ao contrário do tabaco, em que está bem estabelecido que não há níveis considerados "seguros" de consumo, no caso do álcool, nem sempre o consumo pode ser considerado como comportamento de risco, havendo evidências que indicam que o álcool, quando consumido com moderação e regularidade e não combinado a outros comportamentos de risco, como dirigir veículos, pode ter efeitos benéficos em alguns indicadores de saúde 36,37.

A mudança positiva no tabagismo foi associada à incidência de APPS, resultado também encontrado no estudo longitudinal de Sargent-Cox et al. ${ }^{20}$. A relação entre tabagismo e pior autopercepção de saúde também foi verificada em estudos transversais 1,25. O tabagismo é responsável por inúmeros problemas de saúde, e é classificado como um dos quatro principais fatores responsáveis pelo desenvolvimento das doenças crônicas não transmissíveis 11. Deixar de fumar está associado à melhora do condicionamento cardiorrespiratório e vascular, melhora da saúde bucal, entre outros 38 . Os resultados deste estudo sugerem a conotação social negativa que o cigarro vem apresentando, com a reorientação das antigas concepções positivas acerca do uso, o que demonstra efetividade das campanhas públicas de cessação do tabagismo 19. Dessa forma, entende-se que a compreensão entre o hábito de fumar e os prejuízos à saúde já esteja bem estabelecida entre as pessoas. Por outro lado, não foram encontradas associações entre deixar de ser tabagista e a APNS, assim como entre passar a ser tabagista e a APPS. Um dos possíveis motivos para esse resultado é o pequeno número de sujeitos que passou a fumar entre o baseline e o seguimento (apenas sete indivíduos).

Este estudo apresenta alguns pontos positivos e também limitações que devem ser consideradas. Destaca-se positivamente o desenho longitudinal, possibilitando compreender a relação causal em termos temporais entre os comportamentos relacionados à saúde e a autopercepção de saúde. Também se destaca o fato de as análises ajustadas, para algumas variáveis, considerarem a mudança da condição inicial e não meramente a condição do baseline. Assim, diminuiu-se a chance de que outras mudanças (por exemplo, no nível econômico e no estado nutricional), que estão teoricamente associadas tanto aos comportamentos quanto à autopercepção de saúde, confundissem os achados do estudo.

Por outro lado, não houve controle temporal dos acontecimentos relativos aos comportamentos relacionados à saúde, não sendo possível estabelecer o momento exato em que essas mudanças aconteceram, nem sequer se pode ter certeza de que essas alterações sejam permanentes. No entanto, essas limitações são muito difíceis de serem superadas em estudos de coorte de base populacional. Somente se houvesse um acompanhamento mais frequente é que essa limitação poderia ser minimizada. Todavia, esse acompanhamento mais frequente poderia gerar outras limitações, como o próprio princípio de um estudo de coorte ser observacional e não experimental. Outra limitação a ser considerada é o baixo poder de algumas análises, especialmente as relativas ao tabagismo e ao consumo abusivo de álcool, em função do pequeno número de pessoas que passou a apresentar esses comportamentos de risco entre o baseline e o seguimento. Isso pode ter comprometido o poder das análises, não se observando associações significativas, mesmo quando o valor do risco relativo foi elevado. Ainda com relação à questão do poder das análises, a escolha pela estratificação das análises com base na 
condição inicial de cada variável independente também é um ponto a ser observado. Porém, considerando o objetivo do estudo, esta foi a alternativa mais adequada para estimar os efeitos da mudança dos comportamentos em saúde na mudança da autopercepção de saúde. Por fim, é necessário chamar a atenção para as perdas não aleatórias no seguimento, especialmente relativa aos comportamentos relacionados à saúde, pois em geral observaram-se maiores perdas de seguimento entre aqueles que tinham comportamentos negativos no baseline. Essa perda de acompanhamento é considerada um potencial viés de seleção, sendo necessário ter cautela na extrapolação dos resultados. Esse é mais um fator que pode diminuir o poder das análises, especialmente nos grupos em que aconteceu maior percentual de perdas.

Considerando os resultados deste estudo, bem como suas limitações, conclui-se que algumas mudanças nos comportamentos relacionados à saúde podem influenciar a mudança na autopercepção de saúde. Isso ficou mais evidente nas mudanças consideradas negativas na exposição aos comportamentos, especificamente no caso da atividade física no tempo livre e consumo de frutas hortaliças, pois aqueles que deixaram de ter o comportamento considerado positivo em relação à saúde tiveram maior incidência de APNS, se comparados aos que se mantiveram ativos fisicamente no tempo livre ou com consumo regular de frutas e hortaliças. Por outro lado, passar a ser ativo fisicamente no tempo livre ou a consumir frutas e hortaliças de maneira regular não se mostrou associado à mudança na autopercepção de saúde. Já no caso do tabaco, observou-se que deixar de fumar esteve relacionado ao aumento da APPS. Recomenda-se maior número de pesquisas longitudinais que explorem a associação entre a modificação nos comportamentos relacionados à saúde e as mudanças na autopercepção de saúde, examinando essa relação em diferentes populações, faixas etárias e gêneros, com análise combinada de comportamentos e pesquisas que incluam outros indicadores de saúde, a fim de se compreender melhor os fatores que influenciam a autopercepção de saúde.

\section{Colaboradores}

G. F. Andrade contribuiu substancialmente para a concepção e delineamento do estudo, análise e interpretação dos dados do trabalho, bem como elaboração de versões preliminares do artigo e aprovação final da versão a ser publicada. M. R. Loch e A. M. R. Silva contribuíram substancialmente para a concepção do estudo, revisão crítica de importante conteúdo intelectual e aprovação final da versão a ser publicada.

\section{Informações adicionais}

ORCID: Giovana Frazon de Andrade (0000-00029120-0600); Mathias Roberto Loch (0000-00022680-4686); Ana Maria Rigo Silva (0000-00029527-4914).

\section{Agradecimentos}

Agradecimentos ao Conselho Nacional de Desenvolvimento Científico e Tecnológico (CNPq), pelo apoio financeiro, e à Coordenação de Aperfeiçoamento de Pessoal de Nível Superior (Capes), pela concessão de bolsa para realização desta pesquisa. 


\section{Referências}

1. Peres MA, Masiero AV, Longo GZ, Rocha GC, Matos IB, Najnie K, et al. Auto-avaliação da saúde em adultos no Sul do Brasil. Rev Saúde Pública 2010; 44:901-11.

2. Feng Q, Zhu H, Zhen Z, Gu D. Self-rated health, interviewer-rated health, and their predictive powers on mortality in old age. J Gerontol B Psychol Sci Soc Sci 2016; 71:538-50.

3. Moreno X, Huerta M, Albala C. Autopercepción de salud general y mortalidad en adultos mayores. Gac Sanit 2014; 28:246-52.

4. Belém PLO, Melo RLP, Pedroza DF, Menezes TN. Autoavaliação do estado de saúde e fatores associados em idosos cadastrados na Estratégia Saúde da Família de Campina Grande, Paraíba. Rev Bras Geriatr Gerontol (Online) 2015; 19:265-76.

5. Cardoso JDC, Azevedo RCS, Reiners AAO, Louzada CV, Espinosa MM. Autoavaliação de saúde ruim e fatores associados em idosos residentes em zona urbana. Rev Gaúch Enferm 2014; 35:35-41.

6. Meireles AL, Xavier CC, Andrade ACS, Friche AAL, Proietti FA, Caiaffa WT. Self-rated health in urban adults, perceptions of the physical and social environment, and reported comorbidities: The BH Health Study. Cad Saúde Pública 2015; 31 Suppl:S120-35.

7. Pavão ALB, Werneck GL, Campos MR. Autoavaliação do estado de saúde e a associação com fatores sociodemográficos, hábitos de vida e morbidade na população: um inquérito nacional. Cad Saúde Pública 2013; 29:723-34.

8. Reichert FF, Loch MR, Capilheiro MF. Autopercepção de saúde em adolescentes, adultos e idosos/Self-reported health status in adolescents, adults and the elderly. Ciênc Saúde Colet 2012; 17:3353-62.

9. Ministério da Saúde. Política Nacional de Promoção da Saúde. Revisão da Portaria MS/GM no 687, de 30 de março de 2006. Brasília: Ministério da Saúde; 2014.

10. Departamento de Atenção Básica, Secretaria de Atenção a Saúde, Ministério da Saúde. Política Nacional de Atenção Básica. Brasília: Ministério da Saúde; 2012.

11. Departamento de Análise de Situação de Saúde, Secretaria de Vigilância em Saúde, Ministério da Saúde. Plano de Ações Estratégicas para o Enfrentamento das Doenças Crônicas Não Transmissíveis (DCNT) no Brasil 2011-2022. Brasília: Ministério da Saúde; 2011.

12. Ministério da Saúde. Portaria no 2.681, de 7 de novembro de 2013. Redefine o Programa Academia da Saúde no âmbito do Sistema Único de Saúde (SUS). Diário Oficial da União 2013; 8 nov.

13. Kikuchi H, Inoue S, Lee I-M, Odagiri Y, Sawada $\mathrm{N}$, Inoue $\mathrm{M}$, et al. Impact of moderate-intensity and vigorous-intensity physical activity on mortality. Med Sci Sports Exerc 2018; 50:715-21.
14. Rossi A, Dikareva A, Bacon SL, Daskalopoulou SS. The impact of physical activity on mortality in patients with high blood pressure: a systematic review. J Hypertens 2012; 30:1277-88.

15. Zhan J, Liu YJ, Cai LB, Xu FR, Xie T, He QQ. Fruit and vegetable consumption and risk of cardiovascular disease: a meta-analysis of prospective cohort studies. Crit Rev Food Sci Nutr 2017; 57:1650-63.

16. Departamento de Atenção Básica, Secretaria de Atenção a Saúde, Ministério da Saúde. Guia alimentar para a população brasileira. 2a Ed. Brasília: Ministério da Saúde; 2014

17. Munhoz TN, Santos IS, Nunes BP, Mola CL, Silva ICM, Matijasevich A. Tendências de consumo abusivo de álcool nas capitais brasileiras entre os anos de 2006 a 2013: análise das informações do VIGITEL. Cad Saúde Pública 2017; 33:e00104516.

18. Garcia LP, Freitas LRS. Consumo abusivo de álcool no Brasil: resultados da Pesquisa $\mathrm{Na-}$ cional de Saúde 2013. Epidemiol Serv Saúde 2015; 24:227-37.

19. José BPS, Corrêa RA, Malta DC, Passos VMA, França EB, Teixeira RA, et al. Mortalidade e incapacidade por doenças relacionadas à exposição ao tabaco no Brasil, 1990 a 2015. Rev Bras Epidemiol 2017; 20:75-89.

20. Sargent-Cox K, Cherbuin N, Morris L, Butterworth P, Anstey KJ. The effect of health behavior change on self-rated health across the adult life course: a longitudinal cohort study. Prev Med 2014; 58:75-80.

21. Souza RKT, Bortoletto MSS, Loch MR, González AD, Matsuo T, Cabrera MAS, et al. Prevalência de fatores de risco cardiovascular em pessoas com 40 anos ou mais de idade, em Cambé, Paraná (2011): estudo de base populacional. Epidemiol Serv Saúde 2013; 22:435-44.

22. Secretaria de Vigilância em Saúde; Secretaria de Gestão Estratégica e Participativa, Ministério da Saúde. Vigitel Brasil 2008: Vigilância de Fatores de Risco e Proteção para Doenças Crônicas por Inquérito Telefônico. Brasília: Ministério da Saúde; 2010. (Série G. Estatística e Informação em Saúde).

23. Associação Brasileira de Empresas de Pesquisa. Critério de classificação econômica Brasil, 2012. http://www.abep.org/criterio-brasil (acessado em 10/Abr/2017).

24. Departamento de Vigilância de Doenças e Agravos não Transmissíveis e Promoção da Saúde, Secretaria de Vigilância em Saúde, Ministério da Saúde. Vigitel Brasil 2016: Vigilância de fatores de risco e proteção para doenças crônicas por inquérito telefônico. Brasília: Ministério da Saúde; 2017.

25. Campos ACV, Albala C, Lera L, Sánchez H, Vargas AMD, Ferreira EF. Gender differences in predictors of self-rated health among older adults in Brazil and Chile. BMC Public Health 2015; 15:365. 
26. Confortin SC, Giehl MWC, Antes DL, Schneider IJC, d'Orsi E. Autopercepção positiva de saúde em idosos: estudo populacional no Sul do Brasil. Cad Saúde Pública 2015; 31:104960.

27. Loch MR, Souza RKT, Mesas AE, González AD, Rodriguez-Artalejo F. Associação entre capital social a autopercepção de saúde em adultos brasileiros. Rev Saúde Pública 2015; 49:53.

28. Engberg E, Liira H, Kukkonen-Harjula K, From S, Kautiainen H, Pitkälä K, et al. Associations of physical activity with self-rated health and well-being in middle-aged Finnish men Scand J Public Health 2015; 43:190-6.

29. Warbuton DER, Bredin SSD. Reflections on physical activity and health: what should we recommend? Can J Cardiol 2016; 32:495-504.

30. Abuladze L, Kunder N, Lang K, Vaask S. Associations between self-rated health and health behaviour among older adults in Estonia: a cross-sectional analysis. BMJ Open 2017; 7:e013257.

31. Lima RS, Ferreira Neto JAF, Farias RCP. Alimentação, comida e cultura: o exercício da comensalidade. DEMETRA; 2015; 10:507-22.

32. World Health Organization. Health consequences. In: World Health Organization, editor. Global status report on alcohol and health. Geneva: World Health Organization; 2014. p. 45-58.
33. Jernigan D, Noel J, Landon J, Thornton $\mathrm{N}$ Lobstein T. Alcohol marketing and and youth alcohol consumption: a systematic review of longitudinal studies published since 2008. Addiction 2017; 112 Suppl 1:7-20.

34. Ramos EMB, Alves SMC. A oportunidade perdida: a omissão legislativa para as propagandas sobre bebidas alcoólicas. Cadernos Ibero-Americanos de Direito Sanitário 2016; 5:255-9.

35. Minayo MCS, Deslandes SF. A complexidade das relações entre drogas, álcool e violência. Cad Saúde Pública 1998; 14:35-42.

36. Gepner Y, Golan R, Harman-Boehm I, Henkin Y, Schwarzfuchs D, Shelef I, et al. Effects of initiating moderate alcohol intake on cardiometabolic risk in adults with type 2 diabetes. Ann Intern Med 2015; 163:569-79.

37. Tresserra-Rimbau A, Medina-Remón A, Lamuela-Rarantós RM, Bulló M, Dalas-Salvadí J, Corella D, et al. Moderate red wine consuption is associated with a lower prevalence of the metabolic syndrome in the PREDIMED population. Br J Nutr 2015; 113 Suppl 2:S121-30.

38. World Health Organization. Report on the Global Tobacco Epidemic, 2013: enforcing bans on tobacco advertising, promotion and sponsorship. Geneva: World Health Organization; 2013 


\section{Abstract}

Health self-perception is recognized as an interesting health indicator, due to its capacity to predict morbimortality, among others. Studies that investigate its association with health-related behaviors are more recent and, for the most part, crosssectional, which indicates that this relationship may be better understood in longitudinal studies. This article sought to verify the relationship between changes in health-related behaviors and the incidence of positive and negative health selfperception. We carried out a prospective, population-based cohort study with 883 individuals aged 40 years or more interviewed in 2011 and 2015. We verified the incidence of negative and positive health self-perception according to changes in four health-related behaviors: leisure-time physical activity, fruit and vegetable consumption, abusive alcohol consumption and smoking - using crude and adjusted Poisson regression. The incidence of negative health self-perception was of $27.2 \%$, and was higher, after adjusting for demographic, health, and other behavioral variables, among individuals with negative changes in leisure-time physical activity $(R R=1.88 ; 95 \% C I: 1.17-3.05)$ and in fruit and vegetable consumption $(R R=$ 1.95; 95\%CI: 1.15-3.28). The incidence of positive health self-perception was $33.2 \%$ and was higher among those who had a positive change in smoking $(R R=8.37$; 95\%CI: 2.79-25.09) and lower among those who had a negative change in fruit and vegetable consumption $(R R=0.51 ; 95 \% C I: 0.29$ 0.90). We conclude that some changes in healthrelated behaviors have a possible impact on health self-perception, which reinforces the importance of policies related to health-related behaviors, going beyond their relationship with morbimortality.

Life Style; Health Behavior; Self-Assessment; Health Status Indicators; Longitudinal Studies

\section{Resumen}

La autopercepción de salud es reconocida como un interesante indicador de salud, incluso en función de su capacidad predictora de la morbimortalidad. Los estudios que investigan su asociación con comportamientos relacionados con la salud son más recientes, y en su mayoría, transversales, indicando que puede existir una mejor comprensión de esta relación en estudios longitudinales. El objetivo de este artículo fue verificar la relación entre los cambios en los comportamientos relacionados con la salud y la incidencia de autopercepción positiva $y$ negativa de salud. Se realizó un estudio de cohorte prospectivo, de base poblacional, con 883 individuos de 40 años o más, entrevistados en 2011 y 2015. Se verificó la incidencia de autopercepción negativa y positiva de salud, según los cambios en cuatro comportamientos relacionados con la salud-actividad física durante el tiempo libre, consumo de frutas y hortalizas, consumo abusivo de alcohol y tabaquismo- mediante la regresión de Poisson bruta y ajustada. La incidencia de autopercepción negativa de salud fue un $27,2 \%$, siendo mayor, tras los ajustes con variables demográficas, de salud y demás comportamientos, entre los individuos que presentaron un cambio negativo en la actividad física durante el tiempo libre $(R R=$ 1,88; IC95\%: 1,17-3,05) y en el consumo de frutas y hortalizas ( $R R=1,95$; IC95\%: 1,15-3,28). La incidencia de autopercepción positiva de salud fue $33,2 \%$, siendo superior en los que tuvieron cambio positivo en el tabaquismo $(R R=8,37$; IC95\%: 2,79-25,09) e inferior en los que presentaron un cambio negativo en el consumo de frutas y hortalizas $(R R=0,51$; IC95\%: 0,29-0,90). Se concluyó que algunas modificaciones en los comportamientos relacionados con la salud tienen un posible impacto en la autopercepción de la salud, lo que refuerza la importancia de políticas públicas relativas a los comportamientos relacionados con la salud, yendo más allá de su relación con la morbimortalidad.

Estilo de Vida; Conductas Relacionadas con la Salud; Autoevaluación; Indicadores de Salud; Estudios Longitudinales

Recebido em 01/Ago/2018

Versão final reapresentada em 13/Dez/2018

Aprovado em 27/Dez/2018 